\title{
Regulation of alternative splicing by p300-mediated acetylation of splicing factors
}

\author{
AHMAD SIAM, ${ }^{1}$ MAI BAKER, ${ }^{1}$ LEAH AMIT, ${ }^{1}$ GAL REGEV ${ }^{1}$ ALONA RABNER, ${ }^{2}$ RAUF AHMAD NAJAR, ${ }^{1}$ \\ MERCEDES BENTATA, ${ }^{1}$ SARA DAHAN,${ }^{1}$ KLIL COHEN,${ }^{1}$ SARAH ARATEN,${ }^{1}$ YUVAL NEVO,${ }^{1}$ GILLIAN KAY, ${ }^{1}$ \\ YAEL MANDEL-GUTFREUND, ${ }^{2}$ and MAAYAN SALTON ${ }^{1}$ \\ ${ }^{1}$ Department of Biochemistry and Molecular Biology, The Institute for Medical Research Israel-Canada, Faculty of Medicine, The Hebrew \\ University of Jerusalem, Jerusalem 91120, Israel \\ ${ }^{2}$ Faculty of Biology, Technion-Israel Institute of Technology, Haifa 32000, Israel
}

\begin{abstract}
Splicing of precursor mRNA (pre-mRNA) is an important regulatory step in gene expression. Recent evidence points to a regulatory role of chromatin-related proteins in alternative splicing regulation. Using an unbiased approach, we have identified the acetyltransferase $\mathrm{p} 300$ as a key chromatin-related regulator of alternative splicing. p300 promotes genome-wide exon inclusion in both a transcription-dependent and -independent manner. Using CD44 as a paradigm, we found that p300 regulates alternative splicing by modulating the binding of splicing factors to pre-mRNA. Using a tethering strategy, we found that binding of p300 to the CD44 promoter region promotes CD44v exon inclusion independently of RNAPII transcriptional elongation rate. Promoter-bound p300 regulates alternative splicing by acetylating splicing factors, leading to exclusion of hnRNP M from CD44 pre-mRNA and activation of Sam68. p300-mediated CD44 alternative splicing reduces cell motility and promotes epithelial features. Our findings reveal a chromatin-related mechanism of alternative splicing regulation and demonstrate its impact on cellular function.
\end{abstract}

Keywords: pre-mRNA splicing; alternative splicing; chromatin

\section{INTRODUCTION}

Splicing of precursor mRNA (pre-mRNA) is a critical regulatory step in gene expression. The process of alternative splicing enables the production of multiple protein isoforms from a single pre-mRNA molecule by combinatorial use of splice sites, thereby contributing significantly to proteomic diversity (Yang et al. 2016). Despite its undisputed importance in physiological and pathological processes, the question of how alternative splicing decisions are made and how alternative splicing patterns are established and maintained in a cell type- and tissue-specific fashion remains poorly understood.

Studies of alternative splicing have traditionally focused on regulatory elements in the pre-mRNA. Recent work has begun to uncover an additional layer of RNA splicing regulation by demonstrating a strong influence of chromatin structure and epigenetic modifications on splicing regulation (Schwartz and Ast 2010; Hnilicová and Staněk 2011; Luco et al. 2011; de Almeida and Carmo-Fonseca 2014; Haque and Oberdoerffer 2014; Nieto Moreno et al. 2015). These findings raise the intriguing possibility that

Corresponding author: maayan.salton@mail.huji.ac.il

Article is online at http://www.rnajournal.org/cgi/doi/10.1261/rna. 069856.118. epigenetic mechanisms not only control gene transcription but also determine alternative pre-mRNA splicing patterns. Our earlier work identified novel chromatin-related regulators of alternative splicing (Salton et al. 2014). Among these, the acetyltransferase p300 was found to promote inclusion of the microtubule-associated protein tau (MAPT) exon 10 dual-color reporter gene in the most profound way (Stoilov et al. 2008; Salton et al. 2014). p300 acetylates histones as well as other proteins such as transcription factors and thus has a central role as a transcription coactivator (Dancy and Cole 2015).

A strong connection between transcription and splicing has been known for 30 yr (Cramer et al. 1997). Switching a gene's promoter or adding an RNA polymerase II (RNAPII) pause site in a gene body can alter alternative splicing of a specific gene (Cramer et al. 1997; Roberts et al. 1998). In addition, slow kinetics of RNAPII elongation promotes exon inclusion; the included exon is the first to transcribe and consequently the first to splice (Naftelberg et al.

(C) 2019 Siam et al. This article is distributed exclusively by the RNA Society for the first 12 months after the full-issue publication date (see http://rnajournal.cshlp.org/site/misc/terms.xhtml). After 12 months, it is available under a Creative Commons License (Attribution-NonCommercial 4.0 International), as described at http:// creativecommons.org/licenses/by-nc/4.0/. 
2015). On the contrary, RNAPII fast kinetics promotes exon skipping as it exposes additional splice sites (Naftelberg et al. 2015).

Chromatin is a major effector of RNAPII kinetics. Nucleosomes are nonrandomly positioned along the genome and serve as speed bumps for RNAPII (Andersson et al. 2009; Tilgner et al. 2009). This direct effect of nucleosomes on RNAPII can dictate the alternative splicing pattern of the gene (Schwartz et al. 2009; Spies et al. 2009). In addition to nucleosome exon marking, modifications on histone tails in alternatively spliced exons can provide the signal for either inclusion or exclusion, for example by serving as docking site for a protein complex called an adaptor system (Kolasinska-Zwierz et al. 2009; Salton et al. 2014). This can promote splicing factor enrichment that will affect the exon inclusion status (Sims et al. 2007; Gunderson and Johnson 2009; Luco et al. 2011; Salton et al. 2014). Another mechanism is by directly affecting nucleosome occupancy; specifically, histone acetylation results in the eviction of ORF nucleosomes during transcription elongation consequently promoting RNAPII fast kinetics (Church and Fleming 2017) and exon exclusion (Schor et al. 2009, 2013; Dušková et al. 2014). In contrast, DNA and histone tail inhibitory methylation has been connected to RNAPII slow kinetics and exon inclusion (Shukla et al. 2011; Agirre et al. 2015; Lev Maor et al. 2015; Yearim et al. 2015; Singh et al. 2017).

To identify P300's mode of action, we conducted a genome-wide investigation in MCF7 cells silenced for p300. Our results demonstrate the dual role of p300 as an important regulator of both transcription and splicing. Importantly, our work revealed that p300's regulation of alternative splicing correlates with a change in expression in only $30 \%$ of its targets. Thus, histone acetylation or even RNAPII carboxy-terminal domain (CTD) acetylation (Schröder et al. 2013) by p300 might not be the only paths by which p300 can regulate alternative splicing.

Using the p300 target CD44 as a paradigm, we show that modulating chromatin at the promoter using the dCas9-p300/CRISPR system (Hilton et al. 2015) regulates its transcription and splicing via distinct mechanisms. We decipher p300's mode of action as an acetyltransferase of splicing factors. Finally, we show that cell mobility is reduced following tethering of p300's enzymatic core to the CD44 promoter region in MCF7 cells. Our findings reveal a novel mechanism through which chromatin-related proteins regulate alternative splicing.

\section{RESULTS}

\section{p300 regulates transcription and alternative splicing}

Our unbiased screen identified p300 acetyltransferase as an important regulator of alternative splicing (Salton et al. 2014). To begin to unravel the mechanism by which p300 regulates alternative splicing, we characterized all p300-dependent expression and alternative splicing events using deep RNA-sequencing at a genome-wide scale in the breast cancer cell line MCF7 using siRNA (Supplemental Fig. S1A). As expected of a coactivator, we detected 5194 genes that are differentially expressed when p300 is silenced (cut-off set at twofold); of these $<10 \%$ were also alternatively spliced. Interestingly, we found a further group of 892 genes that were exclusively alternatively spliced (Fig. 1A). This result suggests that p300's regulation of alternative splicing can be both dependent and independent of transcription.

Out of all the 1369 genes that were differentially spliced, we observed all types of alternative splicing events: skipped exons (SE; 802 genes), alternative $5^{\prime}$ splice site (A5SS; 151 genes), alternative $3^{\prime}$ splice site (A3SS; 162 genes), mutually exclusive exons (MXE; 295 genes) and retained introns ( $\mathrm{Rl} ; 159$ genes). We validated eight out of the 14 genes that were indicated by RNA-seq to be regulated by alternative splicing by p300 (Supplemental Fig. S1B,C). Using the DAVID function annotation tool (DAVID, https ://david.ncifcrf.gov/) (Huang da et al. 2009a,b), we found that the list of differentially expressed genes was enriched for genes with functions in cell-cell adhesion, cell migration, protein polyubiquitination, and phosphorylation (Fig. 1B). The differentially alternative splicing genes were enriched for genes involved in DNA repair and cell cycle, two known roles of p300, ATP binding and cell-cell adhesion (Fig. 1C). This result demonstrates that p300 can acetylate proteins that are important in the DNA repair pathway (Dutto et al. 2017) and also regulate the alternative splicing of genes in this pathway.

We chose to focus on the largest group of alternative splicing events, those in which exons were skipped. Within this group of 802 genes, 310 were included exons (SE_up) and 527 were excluded exons (SE_down) (Supplemental Fig. S1D-F), suggesting that p300 has a slight preference for exon inclusion. Among the exon inclusion events, we found an enrichment for DNA repair, ATP binding, cell cycle and cell-cell adhesion (Supplemental Fig. $\mathrm{S} 1 \mathrm{H}$ ). In order to delve into the p300 mechanism, we chose CD44, a gene with a role in cell-cell adhesion from the exon inclusion group, which was the most enriched group in both expression and alternative splicing groups (Supplemental Fig. S1I). CD44 is a cell surface glycoprotein involved in cell-cell interactions, cell adhesion and migration. Our deep-sequencing results show that silencing p300 promotes exclusion of CD44v (Supplemental Fig. S2A-C).

\section{p300 up-regulates $C D 44$ transcription and promotes CD44v exon inclusion}

CD44 contains nine variable exons located between constitutive exons 5 and 6 (Supplemental Fig. S2C). The 

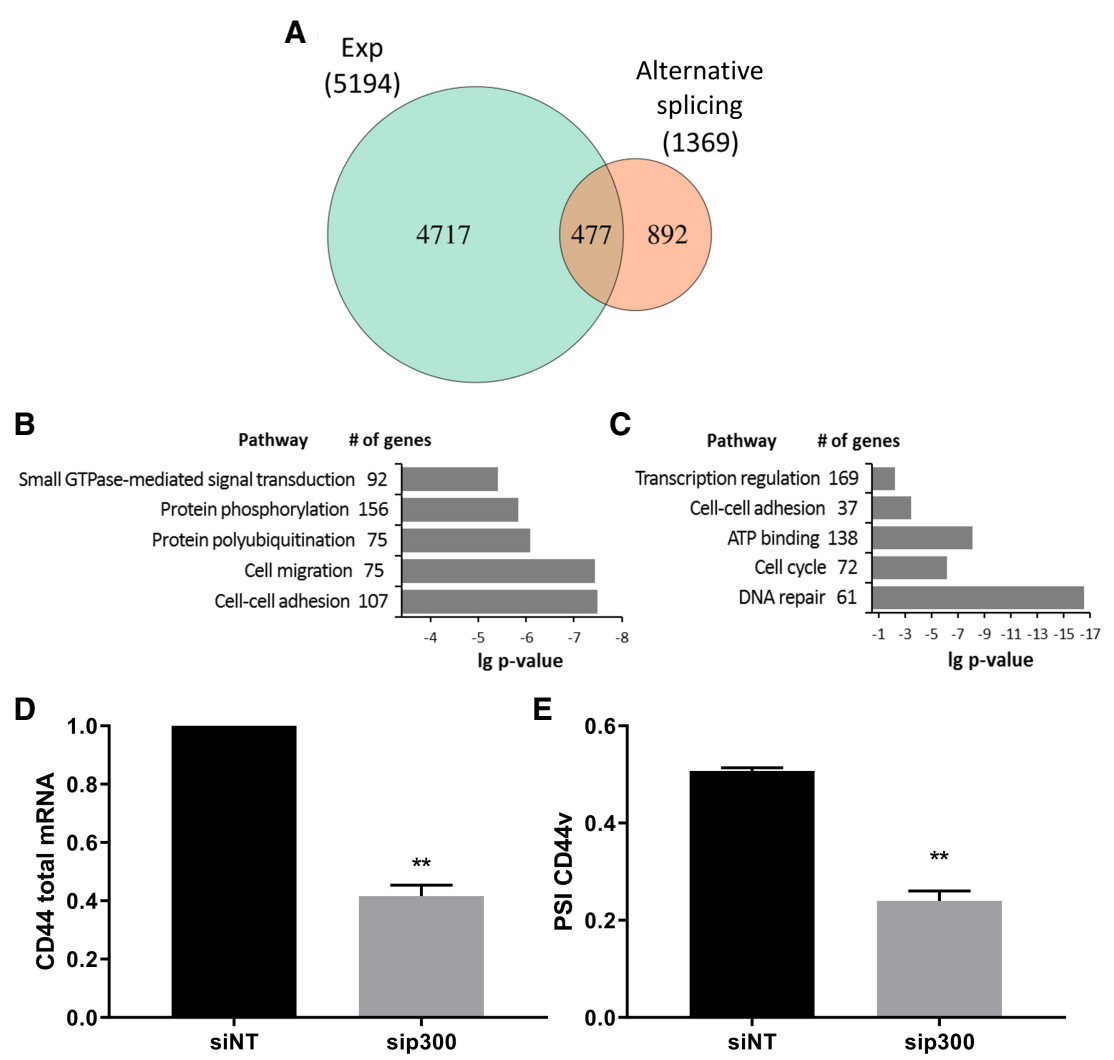

FIGURE 1. $p 300$ is a regulator of both transcription and alternative splicing. MCF7 cells were transfected with nontargeting siRNA (siNT) and sip300 for $72 \mathrm{~h}$. RNA was extracted, libraries prepared, and RNA-seq was conducted. (A) Venn diagram representing differentially expressed genes (light green) and alternatively spliced genes (light orange). (B,C) Functional analysis was conducted using the DAVID functional annotation tool (DAVID, https://david .ncifcrf.gov/) for $(B)$ differentially expressed genes and $(C)$ alternative splicing genes. (D) Real-time PCR analysis of total mRNA amount of CD44 relative to CycloA reference gene. (E) Real-time PCR analysis of CD44s and CD44v5-6 relative to CD44 total mRNA amount. PSI was calculated by CD44v5-6/CD44s + CD44v5-6. Values represent averages of three independent experiments $\left.\pm \mathrm{SE} ;{ }^{(* *}\right) P<0.01$ (paired Student's t-test).

variable exons encode for the extracellular domain of CD44. The standard form of CD44, which excludes all variable exons, is important in epithelial-mesenchymal transition (EMT) (Brown et al. 2011; Xu et al. 2014). Our genome-wide investigation indicated that CD44's transcription and alternative splicing are regulated by p300. In agreement with the RNA-seq results, targeted analysis of CD44 showed that p300 silencing resulted in a lower amount of total CD44, as expected when silencing a coactivator, and CD44v exon exclusion (more CD44s, the short version of CD44, and less CD44v5-6) (Fig. 1D,E).

We began our mechanistic investigation by asking whether p300's effect on CD44 alternative splicing is direct, by changing chromatin in CD44's locus, or indirect, possibly by changing expression of a splicing factor. We set up a system to manipulate chromatin at a specific region of the CD44 gene. To this end, we used a well-characterized nuclease-deficient Cas9 (dCas9) conjugated to the p300 enzymatic core (Hilton et al. 2015). The CRISPR/
dCas9 system allows us to tether a chromatin protein core domain to specific chromosome locations using a pool of four gRNAs. A pool of gRNAs was shown empirically to perform better than a single gRNA (Hilton et al. 2015). We chose HEK293T cells for their low expression of CD44 compared to MCF7, contributing to a high dynamic range when tethering p300 core to the promoter. We cotransfected HEK293T cells with dCas9p300 core (mut) or dCas9-p300 core (WT) and four gRNA plasmids targeting either CD44 promoter, intron 8, or the gene's 3' UTR (Supplemental Fig. S3A). Our results demonstrate that tethering $\mathrm{p} 300$ 's enzymatic core to the CD44 promoter region upregulates CD44 total mRNA fivefold (Fig. 2A). Furthermore, inclusion of CD44 variable exons was promoted (Fig. 2B; Supplemental Fig. S3B,C). Our results show that tethering p300 to the intragenic region $(\ln 8)$ or at the 3' UTR did not affect either transcription or alternative splicing.

ChIP analysis using $\mathrm{H} 3$ and $\mathrm{H} 3$ pan acetylation ( $\mathrm{H} 3$ panAce) antibody showed a significant enrichment in $\mathrm{H} 3$ panAce at the tethered region but not in any other region in the gene (Fig. 2C; Supplemental Fig. S3D), suggesting that histone acetylation induced at a specific region does not spread to the intragenic region. In addition, chromatin immunoprecipitation (ChIP) analysis using RNAPII phospho-serine 5 (pSer5 RNAPII) showed a significant enrichment at the promoter region but not in any other region in the gene when tethering dCas9-p300 core (WT) to CD44 promoter region (Supplemental Fig. S4A,B). This suggests that p300 acetylation of histones as well as RNAPII CTD promotes transcription initiation and thus occupancy of pSer5 RNAPII.

\section{RNAPII elongation rate does not regulate alternative splicing of CD44}

p300 acetylates histones to open chromatin and in addition acetylates RNAPII CTD tail to allow transcription of a subset of genes (Schröder et al. 2013). To examine the role of RNAPII elongation rate in the alternative splicing of CD44, we cotransfected HEK293T cells with plasmids expressing $\alpha$-amanitin resistant polymerases: a mutant "slow" RNAPII (mutant C4) or WT RNAPII (de la Mata 

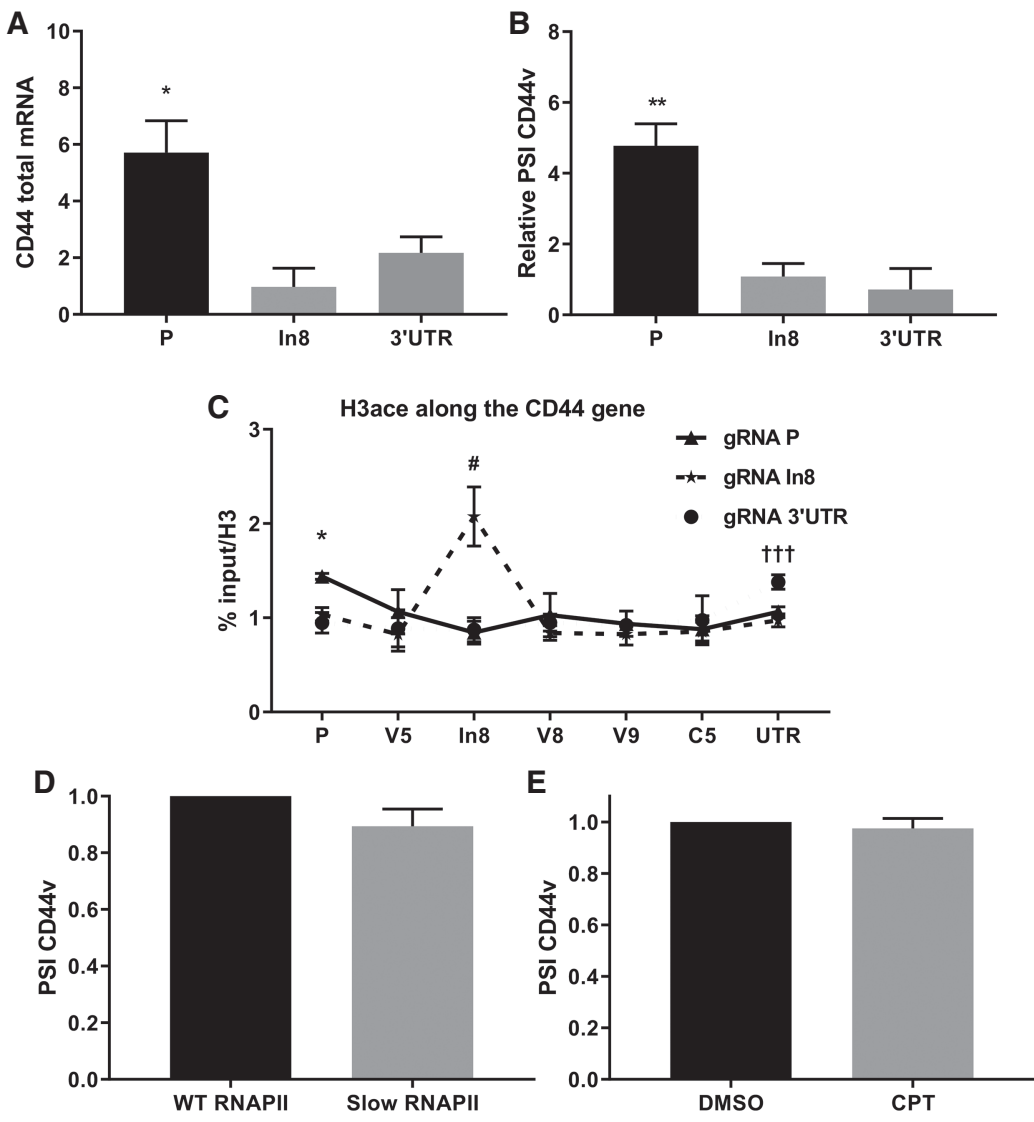

FIGURE 2. Tethering p300 to CD44 promoter up-regulates its expression and promotes CD44v inclusion. (A-C) HEK293T cells were transfected with either dCas9-p300 core (mut) or dCas9-p300 core (WT) with four gRNAs targeted to the CD44 promoter, Intron 8 (In8) or 3' UTR for $30 \mathrm{~h}$. Total RNA was extracted and analyzed by real-time PCR for total mRNA amount of CD44 relative to CycloA reference gene $(A)$ and for total mRNA amount of CD44s and CD44v6-7 relative to CD44 total mRNA amount (B). PSI was calculated by CD44v6-7/CD44s + CD44v6-7. ChIP was performed of H3 pan-acetylated, along the CD44 gene $(C)$. Values represent averages of three independent experiments $\pm \mathrm{SE}$ and are expressed as dCas9-p300 core (WT) relative to dCas9-p300 core (mut) $\left(^{*}\right) P<0.05,(* *) P<$ 0.01, for gRNA to promoter; (\#) $P<0.05$ gRNA to In8; (†††) $P<0.005$ gRNA to $3^{\prime}$ UTR; Student's paired $t$-test. $(D, E)$ HEK293T cells were transfected with either WT RNAPII or Slow RNAPII and after $24 \mathrm{~h}$ treated with $\alpha$-amanitin for $24 \mathrm{~h}(D)$; or cells were treated with $6 \mu \mathrm{M}$ $\mathrm{CPT}$ for $6 \mathrm{~h}(E)$. Total RNA was extracted and analyzed by real-time PCR for CD44s and CD44v6-7 relative to CD44 total mRNA amount. PSI was calculated by CD44v6-7/CD44s + CD44v6-7. Values represent mean \pm SE of seven $(D)$ or three $(E)$ independent experiments. and alternative splicing of two targets of p300 (Supplemental Fig. S4G,H). These findings suggest that CD44 alternative splicing is not affected by RNAPII kinetics. In short, RNAPII kinetics do not mediate the alternative splicing of CD44 suggesting that p300's mechanism of action involves additional factors.

\section{hnRNP M and Sam68 are acetylated in a p300-dependent manner}

Our results led us to speculate that p300 acetylates additional factors that are involved in the regulation of CD44. In order to search for such factors, we asked which of CD44's known splicing factors, TRA2 $\beta$, Sam68, SRm160, hnRNP L, ESRP1, hnRNP M, hnRNP $F$, and hnRNP $A 1$, is enriched in p300 skipped exons (Stoss et al. 2001; Cheng and Sharp 2006; Watermann et al. 2006; Brown et al. 2011; Cappellari et al. 2014; Xu et al. 2014; Loh et al. 2015a,b). From the list of six splicing factors with a known binding motif, we found Sam68 and hnRNP $M$ to be enriched (Fig. 3A). While Sam68 promotes CD44v exon inclusion, mimicking the effect of p300, hnRNP M promotes exclusion. Previous work on Sam68 has shown that it is acetylated by CREB-binding protein (CBP), which is a p300 paralog, resulting in its activation in cancer cells (Babic et al. 2004). A detailed investigation suggested another acetyltransferase is responsible for its acetylation in several cell lines (Babic et al. 2004). In addition, p300 acetylome was reet al. 2003). $\alpha$-amanitin blocks only endogenous RNAPII transcription as the WT and "slow" plasmids are resistant to it. We assessed the alternative splicing pattern of CD44 generated by transcription with the recombinant polymerases. As expected based on previous studies (de la Mata et al. 2003), total CD44 mRNA was lower (Supplemental Fig. S4C); however, we did not observe any change in alternative splicing of CD44 under these conditions (Fig. 2D). In addition, we have slowed RNAPII by using a low dose of CPT $(6 \mu \mathrm{M})$ in both MCF7 and 293T cells. Neither transcription nor splicing alterations of CD44 were observed (Fig. 2E; Supplemental Fig. S4D-F). As a control for the CPT method, we show a change of transcription cently published; in the long list of acetylated proteins we found many splicing factors including Sam68 and hnRNP $M$, both acetylated on several lysines (Weinert et al. 2018). In order to validate that Sam68 is acetylated by p300, we silenced p300 in HEK293T cells and immunoprecipitated Sam68 (Supplemental Fig. S5A). Detection with an antibody against lysine acetylation (KA) demonstrated acetylation of Sam68 (Supplemental Fig. S5B,C) that is dependent on p300 (Fig. 3B; Supplemental Fig. S5D). We achieved similar results immunoprecipitating hnRNP $M$ and detecting with an antibody against $K A$, suggesting that hnRNP M is also acetylated by p300 (Fig. 3C; Supplemental Fig. S5E-G). 


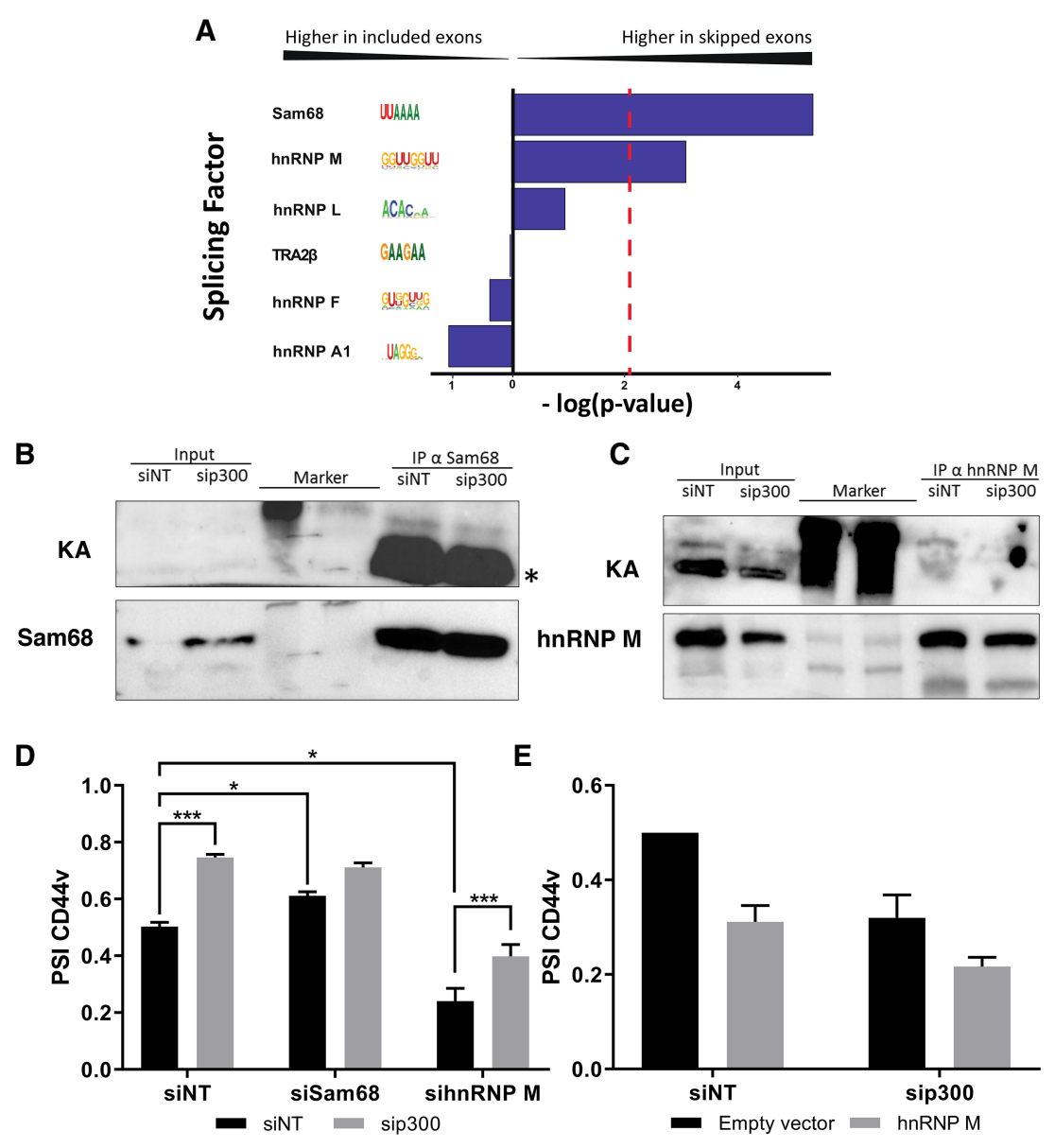

FIGURE 3. p300 acetylates Sam68 and hnRNP M. (A) Enrichment of binding sites of six of CD44's known splicing factors in p300 regulated exons: SAM68, hnRNP $M$, hnRNP L, TRA $2 \beta$, hnRNP $F$, hnRNP A1. Shown are the $-\lg$ ( $P$-values) enrichment of binding sites in skipped exons versus included (right bars) and included versus skipped (left bars). Dashed red line denotes significant $P$-value (corrected for multiple testing, Bonferroni correction). As can be seen, the binding sites of SAM 68 and hnRNP M were found to be significantly enriched in the skipped exons. $(B, C)$ HEK293T cells were transfected with nontargeting siRNA (siNT) and sip300 for $72 \mathrm{~h}$. Sam68 was immunoprecipitated and acetylated lysine (AK) was detected (B). $\left(^{*}\right)$ Heavy chain of Sam68 Ab. hnRNP M was immunoprecipitated and acetylated lysine (KA) was detected $(C)$. (D) MCF7 cells were transfected with nontargeting siRNA (siNT) or siRNA against p300 (sip300) or against Sam68 (siSam68) or hnRNP M (sihnRNP M) for $72 \mathrm{~h}$. Total RNA was extracted and analyzed by real-time PCR for CD44s and CD44v5-6 relative to CD44 total mRNA amount. PSI was calculated by CD44v5-6/CD44s + CD44v5-6. Values represent averages of three independent experiments \pm SE. (*) $P<0.05$; (***) $P<0.001$; Student's paired $t$-test. (E) HEK293T transfected with nontargeting siRNA (siNT) or siRNA against p300 (sip300) and transfected again after $48 \mathrm{~h}$ with either pCDNA3-empty vector or hnRNP M for $24 \mathrm{~h}$. Total RNA was extracted and analyzed by real-time PCR for total mRNA amount of CD44s and CD44v6-7 relative to CD44 total mRNA amount. PSI was calculated by CD44v6-7/CD44s + CD44v6-7. Values represent averages of three independent experiments $\pm \mathrm{SE}$. exon inclusion and hnRNP $M$ promotes exclusion (Fig. 3D). Silencing both p300 and Sam68 gave the same result as silencing either one of them, whereas silencing both p300 and hnRNP M canceled the effect of each protein alone on CD44 alternative splicing (Fig. 3D). Thus, our results suggest that p300 functionally interacts with both Sam68 and hnRNP M.

In order to learn about the relationship between p300 and hnRNP M, we silenced p300 using siRNA and overexpressed hnRNP M in HEK293T cells. We found that p300 silencing and overexpression of hnRNP $M$ resulted in the same extent of CD44v exclusion (Fig. 3E; Supplemental Fig. S6B,C). Moreover, when both p300 is silenced and hnRNP $M$ is over-expressed the effect was additive (Fig. 3E). This result led us to hypothesize that p300 and hnRNP M functionally interact to regulate CD44 alternative splicing.

\section{hnRNP $M$ binding to CD44 pre- mRNA is dependent on $\mathrm{p} 300$}

hnRNP $M$ is known to bind intron 8 of the CD44 pre-mRNA (Xu et al. 2014). To check whether p300 modifies this binding, we performed RNA-IP for hnRNP $M$ following transfection of HEK293T cells with dCas9-p300 core (mut) or dCas9-p300 core (WT) with four gRNA plasmids targeting the CD44 promoter and monitored the CD44 intron 8 region. Tethering the p300 enzymatic core to the CD44 promoter region led to reduction of $\sim 75 \%$ in hnRNP M binding to CD44 intron 8 as compared to CD44 exon 1 (Fig. 4A; Supplemental Fig. S7A,B). This result supports the hypothesis that the two proteins functionally interact in CD44 alternative splicing regulation.

\section{p300 functionally interacts with Sam68 and hnRNP M}

We hypothesized that splicing factor acetylation by p300 will cooperate or hinder p300 in alternative splicing regulation of CD44. In order to test this hypothesis, we silenced either p300 alone or with hnRNP M or Sam68 using siRNA in MCF7 cells (Supplemental Fig. S6A). Our results correspond to the literature whereby Sam68 promotes CD44v

\section{hnRNP M binds HDAC1 to promote CD44v exon exclusion}

Mass spectrometry of endogenous HDAC1 and HDAC2 identified many splicing factors among their interacting partners, including hnRNP M (Khan et al. 2014). Binding of histone deacetylases to hnRNP $M$ would suggest that this is the competing pathway for hnRNP $M$ activation 


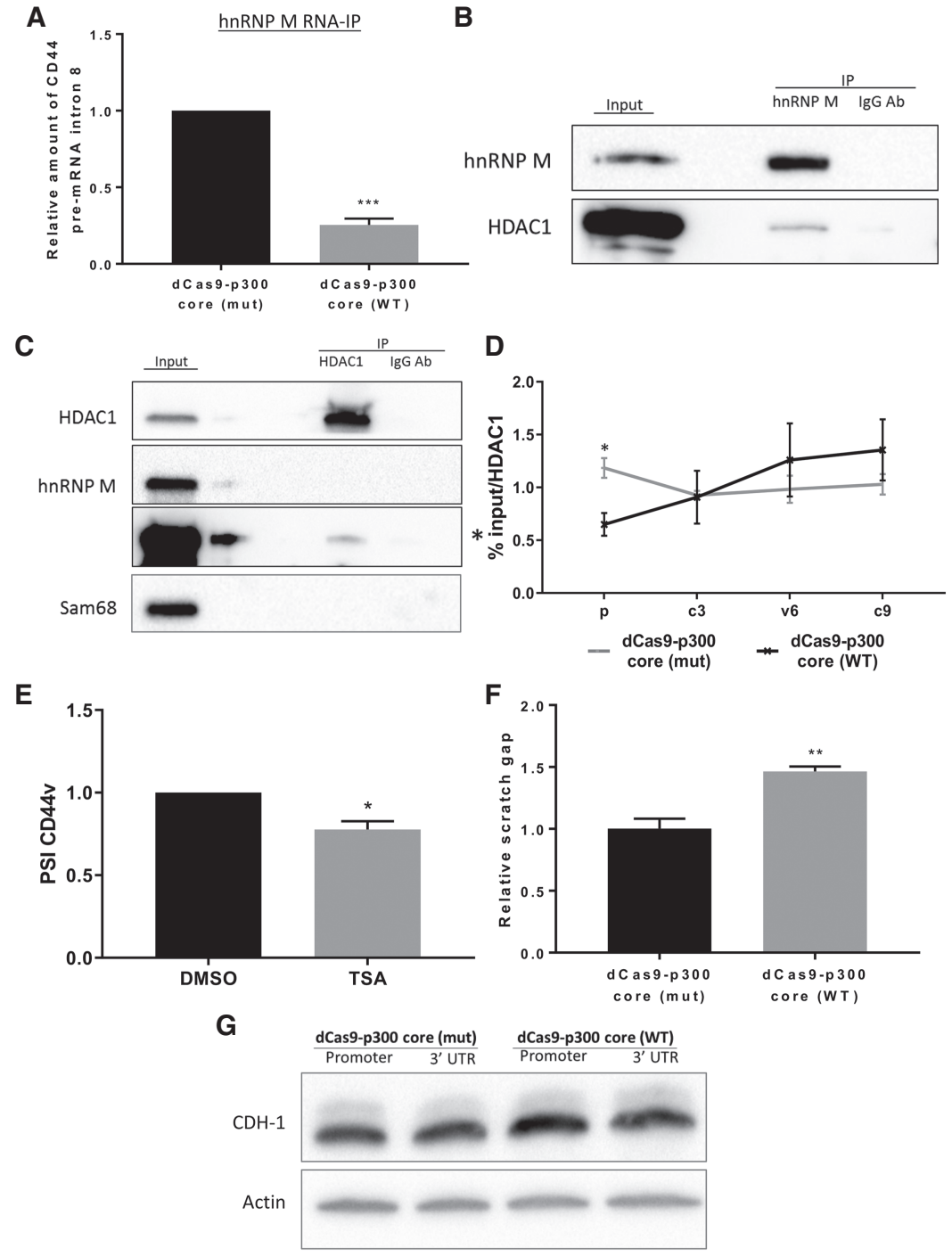

FIGURE 4. p300 excludes hnRNP M from CD44 pre-mRNA and promotes cell motility. (A) RNA-IP of hnRNP $M$ in HEK293T cells transfected with either dCas9-p300 core (mut) or dCas9-p300 core (WT) with four gRNAs targeted to the CD44 promoter. Real-time PCR analysis of CD44 intron 8. Values represent averages of three independent experiments \pm SE. (B) hnRNP M was immunoprecipitated from HEK293T cells and interacting proteins were detected with indicated antibodies. (C) HDAC1 was immunoprecipitated from HEK293T cells and interacting proteins were detected with indicated antibodies. (*) Long exposure. (D) HEK293T cells were transfected with either dCas9-p300 core (mut) or dCas9-p300 core (WT) with four gRNAs targeted to the CD44 promoter for $30 \mathrm{~h}$. ChIP was performed on HDAC1, along the CD44 gene. (E) HEK293T cells were treated with $10 \mu \mathrm{M}$ TSA for $2 \mathrm{~h}$. Real-time PCR analysis of CD44s and CD44v5-6 relative to CD44 total mRNA amount. PSI was calculated by CD44v5-6/CD44s + CD44v5-6. (F,G) MCF7 cells stable for dCas9-p300 core (mut) or dCas9p300 core (WT) were transfected with four gRNAs targeted to CD44 promoter or 3' UTR for $30 \mathrm{~h}$. Cells were seeded in 96-well plates and a scratch assay was conducted. Results were calculated for gRNA to promoter relative to 3' UTR. Values represent averages of three independent experiments \pm SE. $(G)$ Western blot was conducted using the indicated antibodies. endogenous proteins demonstrated physical interaction of HDAC1 with hnRNP $M$ but not with Sam68 (Fig. 4B,C), indicating the presence of these two proteins in the same complex.

\section{Binding of p300 to CD44 promoter excludes HDAC1 binding in this region}

Our assumption is that p300 binds to the CD44 promoter region and acetylates Sam68 and hnRNP M to promote CD44v inclusion. In contrast, binding of HDAC to the region will deacetylate the two splicing factors to promote exon exclusion. To test this hypothesis, we conducted ChIP analysis using HDAC1, which showed a significant reduction at the promoter region but not in any other region in the gene when tethering dCas9-p300 core (WT) to CD44 promoter region (Fig. 4D). This result suggests a binding competition between the complexes of p300 and HDAC1 as was shown before. In addition, we silenced HDAC1 in HEK293T cells and immunoprecipitated hnRNP $M$. Detection with an antibody against lysine acetylation (KA) demonstrated less acetylation of hnRNP $M$ following silencing of HDAC1 (Supplemental Fig. S7C). Finally, treatment of HEK293T cells with Trichostatin A (TSA), a general HDAC inhibitor, mimicked tethering of p300 to up-regulate CD44 total mRNA and promote CD44v exon inclusion (Fig. 4E; Supplemental Fig. S7D).

\section{p300-dependent CD44 alternative splicing affects cell motility and mesenchymal features}

Tethering p300 core can promote a chromatin change only when the region is not predominantly acetylated. HEK293T cells express low amounts since we know that p300 and HDAC complexes compete for DNA binding regions ( $\mathrm{Li}$ et al. 2014). Therefore, we decided to examine if there is physical interaction between hnRNP M and HDAC1 in HEK293T cells. Immunoprecipitation using specific antibodies against the of CD44 and have low $\mathrm{H} 3$ acetylation as we have shown (Fig. 2C), allowing a significant dynamic range and a fiveto sevenfold increase in CD44 total mRNA upon tethering of p300's enzymatic core to the region (Fig. 2A; Supplemental Figs. S4A, S7A). In contrast, CD44 is highly 
expressed in MCF7 cells. Therefore, MCF7 cells stably expressing dCas9-p300 core (WT) and transfected with a pool of gRNA to the CD44 promoter region show a 1.4fold increase of CD44 total mRNA relative to dCas9p300 core (mut) (Supplemental Fig. S8A). In addition to the moderate increase in transcription, we detected a 1.4-fold increase in exon inclusion using both RT-PCR and semi-gPCR (Supplemental Fig. S8B-D).

CD44 alternative splicing is important for EMT, a process during which epithelial cells lose polarity and develop a mesenchymal phenotype (Yang and Weinberg 2008). Key characteristics of EMT are morphological changes and a switch in expression pattern, leading to the acquirement of a migratory phenotype. CD44 alternative splicing is differentially regulated during EMT, whereby the CD44v exons are excluded leading to a switch to CD44s (Brown et al. 2011). CD44s was shown to play a critical role in EMT as its reduction was demonstrated to halt EMT (Brown et al. 2011). The regulation of CD44 alternative splicing is mediated by hnRNP M promoting CD44s by CD44v exclusion (Xu et al. 2014). hnRNP M is thus essential for EMT and for mesenchymal maintenance (Xu et al. 2014). We have shown here that $\mathrm{p} 300$ hinders hnRNP $M$ premRNA binding to CD44 pre-mRNA (Fig. 4A). This result suggests that tethering $\mathrm{P300}$ 's enzymatic core to the CD44 promoter region would hinder MCF7 cell motility. To this end we conducted a scratch assay with MCF7 cells stably expressing dCas9-p300 core (mut) or dCas9-p300 core (WT) and transfected with a pool of gRNA to the CD44 promoter and to the $3^{\prime}$ UTR as an internal control for the cell line. We measured the gap area after $24 \mathrm{~h}$ and found that cells tethered with dCas9-p300 core (WT) had a 40\% bigger gap than dCas9-p300 core (mut) (Fig. 4F; Supplemental Fig. S8E), higher mRNA and protein level of $\mathrm{CDH} 1$, an epithelial marker, and lower mRNA of VIM, a mesenchymal marker (Fig. 4G; Supplemental Fig. S8F$H)$. Our results suggest that p300 has a critical role in transcription and alternative splicing of CD44, cell motility and epithelial characteristics.

\section{DISCUSSION}

The results of our high-throughput screen prompted us to study the role of p300 in alternative splicing regulation. The majority of chromatin-based splicing modulators that we have described are known transcription regulators (Salton et al. 2014). RNA splicing occurs cotranscriptionally and many studies point to coupling of transcription and splicing both kinetically and physically (Luco et al. 2011). Specifically, the CTD of RNAPII has been implicated in splicing factor recruitment and was demonstrated to be acetylated by p300. However, using the CRISPR/dCas9 tethering system we now show that recruiting the p300 enzymatic core to the intragenic region on the CD44 gene does not affect its alternative splicing (Fig. 2B). We specu- late that binding of $\mathrm{p} 300$ to the promoter region of CD44 changes chromatin to allow binding of activator complexes that eventually recruit RNAPII. Binding of these complexes excludes HDAC1 since the two complexes compete for the same binding sites (Li et al. 2014). Thus, our results demonstrate that intragenic acetylation and RNAPII kinetics do not regulate CD44 alternative splicing directly, in contrast to the findings of previous studies (Banine et al. 2005; Batsché et al. 2006; Saint-André et al. 2011; AmeyarZazoua et al. 2012).

Vast literature exists regarding the role of histone acetylation in alternative splicing at the intragenic region. For example, during neuronal cell differentiation, acetylation promotes skipping of exon 18 of the NCAM gene. This observation is consistent with a mechanism involving the kinetic coupling of splicing and transcription (Schor et al. 2009). A connection between acetylation in intragenic regions and alternative splicing was also reported for the $\mathrm{BCl}-x$ gene where progesterone binding recruits $\mathrm{CBP}$ and GCN5 to promote exon 2a inclusion (Bertucci et al. 2013). HDAC inhibitors, such as TSA, were shown to influence splice site selection (Hnilicová et al. 2011). Gene expression following TSA was either increased or unchanged and in both cases exon inclusion and exclusion events were observed (Hnilicová et al. 2011). Specifically, HDAC inhibitors enhanced histone acetylation at the intragenic region of the MCL1 gene leading to exon 2 exclusion mediated by the splicing factor SRSF1 that was shown to bind HDAC1 and 2 (Khan et al. 2014, 2016). In the CDH1 gene, TSA was found to increase inclusion of the $\mathrm{CDH} 1$ exon 8 (Li et al. 2015). In addition, HDAC inactivation by Hu proteins or calcium increased the rate of RNAPII transcriptional elongation and decreased exon inclusion (Zhou et al. 2011; Sharma et al. 2015). In addition to the role of histone acetylation and deacetylation in alternative splicing regulation, p300 has been shown to specifically regulate the EDB1 exon of the FN1 gene (Dušková et al. 2014). p300 bound the promoter of the FN1 minigene and allowed histone 4 acetylation throughout the construct. This modification resulted in a faster elongation rate by RNAPII that mediated the change in splicing (Dušková et al. 2014). Our results add an additional layer of regulation to acetyltransferases and deacetylases, one that is independent of their effect on the chromatin itself.

Posttranslational modifications such as acetylation, ubiquitination, methylation and phosphorylation of splicing factors are well studied (Matter et al. 2002; Risso et al. 2012; Naro and Sette 2013). These modifications were demonstrated to change the factors' binding sites as well as cellular localization and in this way manipulate alternative splicing patterns. Here we suggest that p300 regulates alternative splicing by acetylating the splicing factors hnRNP M and Sam68 (Fig. 5). Sam68 was previously shown to be acetylated and that this modification activates 


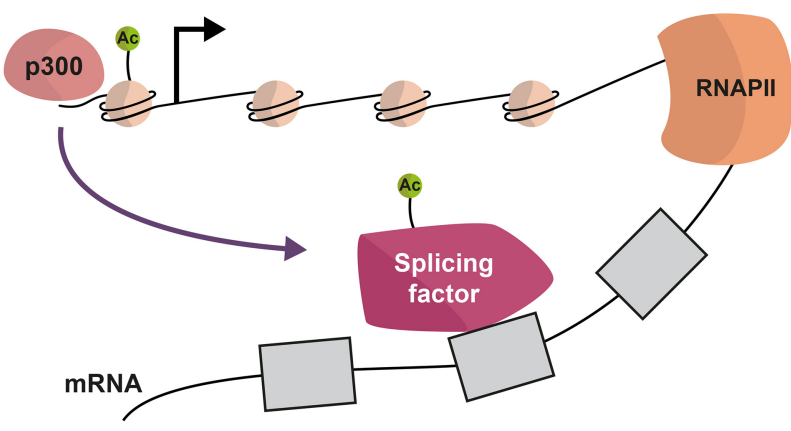

FIGURE 5. Schematic representation of the proposed mechanism. The chromatin factor p300 acetylates histones as well as splicing factors to regulate, respectively, transcription and alternative splicing.

it and is elevated in breast cancer cell lines (Babic et al. 2004; Nakka et al. 2015). Here we found that Sam68 is acetylated in a p300-dependent manner, adding p300 to CBP as known acetyl transferases of Sam68 (Hong et al. 2002). In contrast, acetylation of hnRNP $M$ is described here for the first time and is speculated to reduce its activity. This result establishes a pathway for $\mathrm{p} 300$ as a regulator of splicing factor activity. In addition we demonstrated that hnRNP $M$ is excluded from CD44 pre-mRNA by p300. Furthermore, we located hnRNP $M$ and HDAC1 in the same complex and we hypothesize that when HDAC1 binds to the CD44 promoter, it deacetylates hnRNP M to activate it and promotes exon exclusion the opposite to what we observed when we treated the cells with TSA, which inhibits histone deacetylases (Fig. 4E). In our future work, we intend to specifically map the lysine on Sam68 and hnRNP $M$ that is modified by $\mathrm{p} 300$. In addition, comparing the alternative splicing targets of hnRNP $M$ to those of p300 will allow us to study the magnitude of p300's effect on this splicing factor.

Our results connect the chromatin organization of a gene's promoter to the isoforms generated. This is a new idea in the emerging field linking chromatin organization to changes in alternative splicing. Since p300 also up-regulates the transcription level of a gene, one might predict that the isoforms promoted by p300 are also more potent in nature. Trying to model this hypothesis using CD44 is challenging since CD44 levels were reported to have both positive and negative effects, for example on cancer survival. These contradicting reports stem from a biphasic dependence of the cancer cell on CD44 levels (Klank et al. 2017).

Our results suggest that $\mathrm{p300}$ posttranslationally modifies splicing factors in the vicinity of the CD44 gene or in the CD44 phase separation transcription bubble. The presence of $\mathrm{p} 300$ in the intragenic region $(\ln 8)$ or the UTR did not have the same effect. We predict that it is not p300 levels alone in the phase separation environment but its amount relative to HDAC1 that regulates CD44 alternative splicing.

\section{MATERIALS AND METHODS}

\section{Cell lines and plasmids}

HEK293T (ATCC Number: CRL-3216) and MCF7 (ATCC Number: HTB-22) cells were grown in Dulbecco's modified Eagle's medium (DMEM) supplemented with $10 \%$ fetal bovine serum; cell lines were maintained at $37^{\circ} \mathrm{C}$ and $5 \% \mathrm{CO}_{2}$ atmosphere. Cells were transfected with TransIT-X2 Transfection Reagent (Roche) following the manufacturer's instructions. After $30 \mathrm{~h}$ in culture, plasmid transfected cells were used for experimentation.

pcDNA-dCas9-p300 Core plasmids (D1399Y; plasmid \#61358 and plasmid \#61357) were purchased from Addgene. pSPgRNA (Addgene, plasmid \#47108) was used as the gRNA plasmid. The oligonucleotides containing the target sequences were hybridized, phosphorylated and cloned into the plasmid using Bbsl sites. The target sequences are provided in Supplemental Table S1.

\section{RNAi}

OnTarget Plus SMART pool of four siRNA oligomers per gene against p300, hnRNP M, HDAC1, and Sam68 were purchased from Dharmacon. Cells were grown to $20 \%-50 \%$ confluence and transfected with siRNA using TransIT-X2 transfection reagent. After $72 \mathrm{~h}$ in culture, siRNA transfected cells were used for experimentation.

\section{RNA-seq}

RNA ScreenTape kit (catalog \#5067-5576; Agilent Technologies), D1000 ScreenTape kit (catalog \#5067-5582; Agilent Technologies), Qubit RNA HS Assay kit (catalog \# Q32852; Invitrogen), and Qubit DNA HS Assay kit (catalog \#32854; Invitrogen) were used for each specific step for quality control and quantity determination of RNA and library preparation. For mRNA library preparation: TruSeq RNA Library Prep Kit v2 was used (Illumina). In brief, $1 \mu \mathrm{g}$ was used for the library construction; library was eluted in $20 \mu \mathrm{L}$ of elution buffer. Libraries were adjusted to $10 \mathrm{mM}$, and then $10 \mu \mathrm{L}$ (50\%) from each sample was collected and pooled in one tube. Multiplex sample pool (1.5 pM including PhiX 1.5\%) was loaded in NextSeq 500/550 High Output v2 kit (75 cycles cartridge and 150 cycles cartridge; Illumina). Run conditions were in paired end (43 $\times 43 \mathrm{bp}$ and $80 \times 80 \mathrm{bp}$, respectively) and loaded on NextSeq 500 System machine (Illumina).

We used rMATS (version3.2.5) (Shen et al. 2014) to identify differential alternative splicing events between the two sample groups corresponding to all five basic types of alternative splicing patterns. Briefly, rMATS uses a modified version of the generalized linear mixed model to detect differential alternative splicing from RNA-seq data with replicates, while controlling for changes in overall gene expression levels. It accounts for exon-specific sequencing coverage in individual samples as well as variation in exon splicing levels among replicates. For each alternative splicing event, we used the calculation on both the reads mapped to the splice junctions and the reads mapped to the exon body. We ran rMATS with -c 0.05 parameter (deltaPSI > 0.05\%) and then detected significant splicing events using a cut off at FDR $<5 \%$. 


\section{qRT-PCR}

RNA was isolated from cells using the GENEzol TriRNA Pure Kit (GeneAid). cDNA synthesis was carried out with the Quanta cDNA Reverse Transcription Kit (QuantaBio). qPCR was performed with the iTaq Supermix (BioRad) on the Biorad iCycler. The comparative $\mathrm{Ct}$ method was used to quantify transcripts, and delta Ct was measured in triplicate. Primers used in this study are provided in Supplemental Table S1.

\section{RBP binding analysis}

The occurrence of the binding motifs for six splicing factors: Sam68, hnRNP A1, hnRNP F, hnRNP L, hnRNP M, TRA2 $\beta$ in 555 excluded and 973 included exons was calculated with RBPmap (Paz et al. 2014) using "medium stringency" and "conservation filter" parameters. The number of detected motifs was normalized to the length of each exon. Enrichment of motif occurrences was calculated using the Mann-Whitney Wilcoxon U-test. The Bonferroni method was applied for multiple testing correction. PWMs for the motifs were extracted from RBPmap (hnRNP A1, hnRNP $F$, hnRNP L, hnRNP M) and ATtRACT (TRA2 $\beta$, Sam68) (Giudice et al. 2016).

\section{Chromatin immunoprecipitation (ChIP)}

Approximately $2 \times 10^{6}$ cells per sample were cross-linked for 15 min in $1 \%$ formaldehyde at RT. Cells were washed twice with cold PBS and lysed with lysis buffer (0.5\% sodium dodecyl sulfate [SDS], $10 \mathrm{mM}$ ethylenediaminetetraacetic acid [EDTA], $50 \mathrm{mM}$ Tris- $\mathrm{HCl}, \mathrm{pH} 8$ and $1 \times$ protease inhibitor cocktail). DNA was sonicated in an ultrasonic bath (Qsonica, Q800R2 Sonicator) to an average length of 200-1000 bp. Supernatants were immunoprecipitated overnight with $40 \mu \mathrm{L}$ of precoated anti-IgG magnetic beads (goat anti-rabbit IgG magnetic beads, NEB) previously incubated with the antibody of interest for $6 \mathrm{~h}$ at $4^{\circ} \mathrm{C}$. The antibodies used were: rabbit anti-H3 PanAce (Abcam, cat. no. ab47915) and rabbit anti-RNAPII-ser5 (Abcam, cat. no. ab5131), rabbit anti-histone $\mathrm{H} 3$ (Abcam, cat. no. ab1791), rabbit anti-HDAC1 (Abcam, cat no. ab7028). Beads were washed sequentially for 5 min each in low-salt (20 mM Tris- $\mathrm{HCl} \mathrm{pH} 8$, $150 \mathrm{mM} \mathrm{NaCl}, 2 \mathrm{mM}$ EDTA, 1\% Triton X-100, 0.1\% SDS), high-salt (20 mM Tris- $\mathrm{HCl} \mathrm{pH} 8,500 \mathrm{mM} \mathrm{NaCl}, 2 \mathrm{mM}$ EDTA, $1 \%$ Triton $\mathrm{X}-100,0.1 \% \mathrm{SDS}$ ) and $\mathrm{LiCl}$ buffer $(10 \mathrm{mM}$ Tris $\mathrm{pH}$ 8.0, 1 mM EDTA, $250 \mathrm{mM} \mathrm{LiCl}$, 1\% NP-40, 1\% Na- deoxycholate) and in Tris-EDTA (TE) buffer. Beads were eluted in $1 \%$ SDS and $100 \mathrm{mM} \mathrm{NaHCO} 3$ buffer for $15 \mathrm{~min}$ at $65^{\circ} \mathrm{C}$ and cross-linking was reversed for $6 \mathrm{~h}$ after addition of $\mathrm{NaCl}$ to a final concentration of $200 \mathrm{mM}$ and sequentially treated with $20 \mu \mathrm{g}$ proteinase K. DNA was extracted using magnetic beads (Beckman Coulter, Agencourt AMPure XP, cat. no. A63881). Immunoprecipitated DNA (2 out of $50 \mu \mathrm{L}$ ) and serial dilutions of the $10 \%$ input DNA $(1: 5,1: 25,1: 125$, and 1:625) were analyzed by SYBR-Green real-time qPCR. ChIP-qPCR data were analyzed relative to input to include normalization for both background levels and the amount of input chromatin to be used in ChIP. The primer sequences used are listed in Supplemental Table S1.

\section{RNA-immunoprecipitation (RNA-IP)}

Cells were washed twice with ice-cold PBS, harvested, and lysed for 30 min on ice in a buffer containing 0.5\% NP40 150 mM $\mathrm{NaCl}, 50 \mathrm{mM}$ Tris $\mathrm{pH}=7.5$, and $1 \mathrm{mM}$ EDTA supplemented with protease and RNAsin inhibitors followed by sonication in an ultrasonic bath (Qsonica, Q800R2 Sonicator) for six cycles of 5 sec $O N$ and $30 \mathrm{sec}$ OFF. Supernatants were collected after centrifugation at $21,000 \mathrm{~g}$ for $20 \mathrm{~min}$. Antibody was added for $2 \mathrm{~h}$ at $4^{\circ} \mathrm{C}$. Protein $A$ and $G$ sepharose beads were added for an additional $1 \mathrm{~h}$. Beads were washed four times and GeneZol added for RNA extraction. Serial dilutions of the 10\% input cDNA $(1: 5,1: 25$, 1:125) were analyzed by SYBR-Green real-time GPCR. The oligonucleotide sequences used are listed in Supplemental Table S1.

\section{Immunoprecipitation (IP)}

Cells were washed twice with ice-cold PBS, harvested and lysed for $30 \mathrm{~min}$ on ice in $0.5 \% \mathrm{NP} 40,150 \mathrm{mM} \mathrm{NaCl}, 50 \mathrm{mM}$ Tris $\mathrm{pH}$ 7.5, and $2 \mathrm{mM} \mathrm{MgCl}$ supplemented with protease inhibitor and RNAsin (Hylabs) and for the acetylation investigation with $10 \mu \mathrm{M}$ trichostatin A (TSA) (Frementek). Cell supernatants were collected after centrifugation at $21,000 \mathrm{~g}$ for $20 \mathrm{~min}$. Antibody anti-hnRNP M (4 $\mu$ g, sc-20002), anti-HDAC1 (4 $\mu$ g; ab19845), or Sam68 (4 $\mu$; ab26803) was added for $2 \mathrm{~h}$ at $4^{\circ} \mathrm{C}$. Protein $\mathrm{A}$ and $\mathrm{G}$ sepharose beads were added for an additional $1 \mathrm{~h}$. Beads were washed four times, boiled in sample buffer and loaded onto a $4 \%-12 \%$ Bis-Tris gel, then transferred onto a polyvinylidene difluoride membrane and enhanced chemiluminescence using Clarity Western ECL substrate (Bio-Rad) was used to visualize the protein.

\section{CPT treatment}

To impede the dynamics of transcribing RNAPII, cells were treated with camptothecin (CPT, Sigma) to a final concentration of $6 \mu \mathrm{M}$ for $6 \mathrm{~h}$.

\section{Scratch assay}

Cells were grown to confluence in 96-well plates in DMEM supplemented with $1 \%$ fetal bovine serum. A straight scratch was made using a P20 pipette tip; the gap width was measured and recorded $(0 \mathrm{~h})$ using the ImageJ image processing program. The cells were then incubated for $24 h$, the gap width of scratch repopulation was measured and recorded, and then compared to the initial gap size.

\section{DATA DEPOSITION}

The RNA-seq data has been deposited in the Gene Expression Omnibus (GEO) with the data set identifier GSE109957.

\section{SUPPLEMENTAL MATERIAL}

Supplemental material is available for this article. 


\section{ACKNOWLEDGMENTS}

We thank Chonghui Cheng and Rotem Karni for valuable discussion. We thank Abed Nasereddin and Idit Shiff from the Genomic Applications Laboratory, The Core Research Facility, The Faculty of Medicine - Ein Kerem, The Hebrew University of Jerusalem, Israel, for their professional advice and RNA-seg service. We thank Amina Jbara for guidance on scratch assay technique. We thank Chonghui Cheng for the hnRNP M plasmid and Alberto Kornblihtt for slow RNAPII plasmids. This work was in part supported by the Israeli Cancer Association, Alon Award by the Israeli Planning and Budgeting Committee (PBC), and the Israel Science Foundation (ISF 1154/17). The RNA-seq reagents were funded by a Core Laboratory Grant Program launched by Danyel Biotech, the authorized Illumina Channel Partner in Israel.

Received December 6, 2018; accepted April 8, 2019.

\section{REFERENCES}

Agirre E, Bellora N, Alló M, Pagès A, Bertucci P, Kornblihtt AR, Eyras E. 2015. A chromatin code for alternative splicing involving a putative association between CTCF and HP1 $\alpha$ proteins. BMC Biol 13: 31 . doi:10.1186/s12915-015-0141-5

Ameyar-Zazoua M, Rachez C, Souidi M, Robin P, Fritsch L, Young R, Morozova N, Fenouil R, Descostes N, Andrau JC, et al. 2012. Argonaute proteins couple chromatin silencing to alternative splicing. Nat Struct Mol Biol 19: 998-1004. doi:10.1038/nsmb .2373

Andersson R, Enroth S, Rada-Iglesias A, Wadelius C, Komorowski J. 2009. Nucleosomes are well positioned in exons and carry characteristic histone modifications. Genome Res 19: 1732-1741. doi:10 $.1101 /$ gr.092353.109

Babic I, Jakymiw A, Fujita DJ. 2004. The RNA binding protein Sam68 is acetylated in tumor cell lines, and its acetylation correlates with enhanced RNA binding activity. Oncogene 23: 3781-3789. doi:10 1038/sj.onc. 1207484

Banine F, Bartlett C, Gunawardena R, Muchardt C, Yaniv M, Knudsen ES, Weissman BE, Sherman LS. 2005. SWI/SNF chromatin-remodeling factors induce changes in DNA methylation to promote transcriptional activation. Cancer Res 65: 3542-3547. doi:10 .1158/0008-5472.CAN-04-3554

Batsché E, Yaniv M, Muchardt C. 2006. The human SWI/SNF subunit $\mathrm{Brm}$ is a regulator of alternative splicing. Nat Struct Mol Biol 13: 22-29. doi:10.1038/nsmb1030

Bertucci PY, Nacht AS, Alló M, Rocha-Viegas L, Ballaré C, Soronellas D, Castellano G, Zaurin R, Kornblihtt AR, Beato M, et al. 2013. Progesterone receptor induces bcl-x expression through intragenic binding sites favoring RNA polymerase II elongation. Nucleic Acids Res 41: 6072-6086. doi:10.1093/nar/gkt327

Brown RL, Reinke LM, Damerow MS, Perez D, Chodosh LA, Yang J, Cheng C. 2011. CD44 splice isoform switching in human and mouse epithelium is essential for epithelial-mesenchymal transition and breast cancer progression. J Clin Invest 121: 1064 1074. doi:10.1172/JCl44540

Cappellari M, Bielli P, Paronetto MP, Ciccosanti F, Fimia GM, Saarikettu J, Silvennoinen O, Sette C. 2014. The transcriptional co-activator SND1 is a novel regulator of alternative splicing in prostate cancer cells. Oncogene 33: 3794-3802. doi:10.1038/ onc.2013.360

Cheng C, Sharp PA. 2006. Regulation of CD44 alternative splicing by $\mathrm{SRm} 160$ and its potential role in tumor cell invasion. Mol Cell Biol 26: 362-370. doi:10.1128/MCB.26.1.362-370.2006
Church MC, Fleming AB. 2017. A role for histone acetylation in regulating transcription elongation. Transcription 9: 225-232. doi:10 1080/21541264.2017.1394423

Cramer P, Pesce CG, Baralle FE, Kornblihtt AR. 1997. Functional association between promoter structure and transcript alternative splicing. Proc Natl Acad Sci 94: 11456-11460. doi:10.1073/pnas 94.21.11456

Dancy BM, Cole PA. 2015. Protein lysine acetylation by p300/CBP. Chem Rev 115: 2419-2452. doi:10.1021/cr500452k

de Almeida SF, Carmo-Fonseca M. 2014. Reciprocal regulatory links between cotranscriptional splicing and chromatin. Semin Cell Dev Biol 32: 2-10. doi:10.1016/j.semcdb.2014.03.010

de la Mata M, Alonso CR, Kadener S, Fededa JP, Blaustein M, Pelisch F, Cramer P, Bentley D, Kornblihtt AR. 2003. A slow RNA polymerase $I I$ affects alternative splicing in vivo. Mol Cell 12: 525-532. doi:10.1016/j.molcel.2003.08.001

Dušková E, Hnilicová J, Staněk D. 2014. CRE promoter sites modulate alternative splicing via p300-mediated histone acetylation. RNA Biol 11: 865-874. doi:10.4161/rna.29441

Dutto I, Scalera C, Prosperi E. 2017. CREBBP and p300 lysine acetyl transferases in the DNA damage response. Cell Mol Life Sci 75: 1325-1338. doi:10.1007/s00018-017-2717-4

Giudice G, Sánchez-Cabo F, Torroja C, Lara-Pezzi E. 2016. ATtRACTa database of RNA-binding proteins and associated motifs. Database (Oxford) 2016: baw035. doi:10.1093/database/baw035

Gunderson FQ, Johnson TL. 2009. Acetylation by the transcriptional coactivator Gcn5 plays a novel role in co-transcriptional spliceosome assembly. PLoS Genet 5: e1000682. doi:10.1371/journal .pgen.1000682

Haque N, Oberdoerffer S. 2014. Chromatin and splicing. Methods Mol Biol 1126: 97-113. doi:10.1007/978-1-62703-980-2 7

Hilton IB, D'Ippolito AM, Vockley CM, Thakore PI, Crawford GE, Reddy TE, Gersbach CA. 2015. Epigenome editing by a CRISPRCas9-based acetyltransferase activates genes from promoters and enhancers. Nat Biotechnol 33: 510-517. doi:10.1038/nbt 3199

Hnilicová J, Staněk D. 2011. Where splicing joins chromatin. Nucleus 2: 182-188. doi:10.4161/nucl.2.3.15876

Hnilicová J, Hozeifi S, Dušková E, Icha J, Tománkova T, Staněk D. 2011. Histone deacetylase activity modulates alternative splicing. PLoS One 6: e16727. doi:10.1371/journal.pone.0016727

Hong W, Resnick RJ, Rakowski C, Shalloway D, Taylor SJ, Blobel GA. 2002. Physical and functional interaction between the transcriptional cofactor CBP and the $\mathrm{KH}$ domain protein Sam68. Mol Cancer Res 1: 48-55.

Huang da W, Sherman BT, Lempicki RA. 2009a. Bioinformatics enrichment tools: paths toward the comprehensive functional analysis of large gene lists. Nucleic Acids Res 37: 1-13. doi:10.1093/nar/ gkn923

Huang da W, Sherman BT, Lempicki RA. 2009b. Systematic and integrative analysis of large gene lists using DAVID bioinformatics resources. Nat Protoc 4: 44-57. doi:10.1038/nprot.2008.211

Khan DH, Gonzalez C, Cooper C, Sun JM, Chen HY, Healy S, Xu W, Smith KT, Workman JL, Leygue E, et al. 2014. RNA-dependent dynamic histone acetylation regulates MCL1 alternative splicing. Nucleic Acids Res 42: 1656-1670. doi:10.1093/nar/gkt1134

Khan DH, Gonzalez C, Tailor N, Hamedani MK, Leygue E, Davie JR. 2016. Dynamic histone acetylation of H3K4me3 nucleosome regulates MCL1 pre-mRNA splicing. J Cell Physiol 231: 2196-2204. doi:10.1002/jcp.25337

Klank RL, Decker Grunke SA, Bangasser BL, Forster CL, Price MA, Odde TJ, SantaCruz KS, Rosenfeld SS, Canoll P, Turley EA, et al. 2017. Biphasic dependence of glioma survival and cell migration on CD44 expression level. Cell Rep 18: 23-31. doi:10.1016/j .celrep.2016.12.024 
Kolasinska-Zwierz P, Down T, Latorre I, Liu T, Liu XS, Ahringer J. 2009. Differential chromatin marking of introns and expressed exons by H3K36me3. Nat Genet 41: 376-381. doi:10.1038/ng.322

Lev Maor G, Yearim A, Ast G. 2015. The alternative role of DNA methylation in splicing regulation. Trends Genet 31: 274-280. doi:10 .1016/j.tig.2015.03.002

Li X, Yang H, Huang S, Qiu Y. 2014. Histone deacetylase 1 and p300 can directly associate with chromatin and compete for binding in a mutually exclusive manner. PLoS One 9: e94523. doi:10.1371/jour nal.pone.0094523

Li XW, Shi BY, Yang QL, Wu J, Wu HM, Wang YF, Wu ZJ, Fan YM, Wang YP. 2015. Epigenetic regulation of $\mathrm{CDH} 1$ exon 8 alternative splicing in gastric cancer. BMC Cancer 15: 954. doi:10.1186/ s12885-015-1983-5

Loh TJ, Cho S, Moon H, Jang HN, Williams DR, Jung DW, Kim IC, Ghigna C, Biamonti G, Zheng X, et al. 2015a. hnRNP L inhibits CD44 V10 exon splicing through interacting with its upstream intron. Biochim Biophys Acta 1849: 743-750. doi:10.1016/j .bbagrm.2015.01.004

Loh TJ, Moon H, Cho S, Jang H, Liu YC, Tai H, Jung DW, Williams DR, Kim HR, Shin MG, et al. 2015b. CD44 alternative splicing and hnRNP A1 expression are associated with the metastasis of breast cancer. Oncol Rep 34: 1231-1238. doi:10.3892/or.2015.4110

Luco RF, Alló M, Schor IE, Kornblihtt AR, Misteli T. 2011. Epigenetics in alternative pre-mRNA splicing. Cell 144: 16-26. doi:10.1016/j .cell.2010.11.056

Matter N, Herrlich P, König H. 2002. Signal-dependent regulation of splicing via phosphorylation of Sam68. Nature 420: 691-695. doi:10.1038/nature01153

Naftelberg S, Schor IE, Ast G, Kornblihtt AR. 2015. Regulation of alternative splicing through coupling with transcription and chromatin structure. Annu Rev Biochem 84: 165-198. doi:10.1146/annurevbiochem-060614-034242

Nakka KK, Chaudhary N, Joshi S, Bhat J, Singh K, Chatterjee S, Malhotra R, De A, Santra MK, Dilworth FJ, et al. 2015. Nuclear matrix-associated protein SMAR1 regulates alternative splicing via HDAC6-mediated deacetylation of Sam68. Proc Natl Acad Sci 112: E3374-E3383. doi:10.1073/pnas.1418603112

Naro C, Sette C. 2013. Phosphorylation-mediated regulation of alternative splicing in cancer. Int J Cell Biol 2013: 151839. doi:10 $.1155 / 2013 / 151839$

Nieto Moreno N, Giono LE, Cambindo Botto AE, Muñoz MJ, Kornblihtt AR. 2015. Chromatin, DNA structure and alternative splicing. FEBS Lett 589: 3370-3378. doi:10.1016/j.febslet.2015 .08 .002

Paz I, Kosti I, Ares M Jr, Cline M, Mandel-Gutfreund Y. 2014. RBPmap: a web server for mapping binding sites of RNA-binding proteins. Nucleic Acids Res 42: W361-W367. doi:10.1093/nar/gku406

Risso G, Pelisch F, Quaglino A, Pozzi B, Srebrow A. 2012. Regulating the regulators: serine/arginine-rich proteins under scrutiny. IUBMB Life 64: 809-816. doi:10.1002/iub.1075

Roberts GC, Gooding C, Mak HY, Proudfoot NJ, Smith CW. 1998. Cotranscriptional commitment to alternative splice site selection. Nucleic Acids Res 26: 5568-5572. doi:10.1093/nar/26.24.5568

Saint-André V, Batsché E, Rachez C, Muchardt C. 2011. Histone H3 lysine 9 trimethylation and $\mathrm{HP} 1 \gamma$ favor inclusion of alternative exons. Nat Struct Mol Biol 18: 337-344. doi:10.1038/nsmb.1995

Salton M, Voss TC, Misteli T. 2014. Identification by high-throughput imaging of the histone methyltransferase EHMT2 as an epigenetic regulator of VEGFA alternative splicing. Nucleic Acids Res 42: 13662-13673. doi:10.1093/nar/gku1226

Schor IE, Rascovan N, Pelisch F, Alló M, Kornblihtt AR. 2009. Neuronal cell depolarization induces intragenic chromatin modifications affecting NCAM alternative splicing. Proc Natl Acad Sci 106: 43254330. doi:10.1073/pnas.0810666106
Schor IE, Fiszbein A, Petrillo E, Kornblihtt AR. 2013. Intragenic epigenetic changes modulate NCAM alternative splicing in neuronal differentiation. EMBO J 32: 2264-2274. doi:10.1038/emboj.2013 .167

Schröder S, Herker E, Itzen F, He D, Thomas S, Gilchrist DA, Kaehlcke K, Cho S, Pollard KS, Capra JA, et al. 2013. Acetylation of RNA polymerase II regulates growth-factor-induced gene transcription in mammalian cells. Mol Cell 52: 314-324. doi:10.1016/j .molcel.2013.10.009

Schwartz S, Ast G. 2010. Chromatin density and splicing destiny: on the cross-talk between chromatin structure and splicing. EMBO J 29: 1629-1636. doi:10.1038/emboj.2010.71

Schwartz S, Meshorer E, Ast G. 2009. Chromatin organization marks exon-intron structure. Nat Struct Mol Biol 16: 990-995. doi:10 $.1038 / \mathrm{nsmb} .1659$

Sharma A, Nguyen H, Cai L, Lou H. 2015. Histone hyperacetylation and exon skipping: a calcium-mediated dynamic regulation in cardiomyocytes. Nucleus 6: 273-278. doi:10.1080/19491034.2015 1081324

Shen S, Park JW, Lu ZX, Lin L, Henry MD, Wu YN, Zhou Q, Xing Y. 2014. rMATS: robust and flexible detection of differential alternative splicing from replicate RNA-seq data. Proc Natl Acad Sci 111: E5593-5601. doi:10.1073/pnas.1419161111

Shukla S, Kavak E, Gregory M, Imashimizu M, Shutinoski B, Kashlev M, Oberdoerffer P, Sandberg R, Oberdoerffer S. 2011. CTCF-promoted RNA polymerase II pausing links DNA methylation to splicing. Nature 479: 74-79. doi:10.1038/nature10442

Sims RJ III, Millhouse S, Chen CF, Lewis BA, Erdjument-Bromage $H$, Tempst P, Manley JL, Reinberg D. 2007. Recognition of trimethylated histone $\mathrm{H} 3$ lysine 4 facilitates the recruitment of transcription post-initiation factors and pre-mRNA splicing. Mol Cell 28: 665-676. doi:10.1016/j.molcel.2007.11.010

Singh S, Narayanan SP, Biswas K, Gupta A, Ahuja N, Yadav S, Panday RK, Samaiya A, Sharan SK, Shukla S. 2017. Intragenic DNA methylation and BORIS-mediated cancer-specific splicing contribute to the Warburg effect. Proc Natl Acad Sci 114: 11440-11445. doi:10.1073/pnas.1708447114

Spies N, Nielsen CB, Padgett RA, Burge CB. 2009. Biased chromatin signatures around polyadenylation sites and exons. Mol Cell 36: 245-254. doi:10.1016/j.molcel.2009.10.008

Stoilov P, Lin CH, Damoiseaux R, Nikolic J, Black DL. 2008. A highthroughput screening strategy identifies cardiotonic steroids as alternative splicing modulators. Proc Natl Acad Sci 105: 1121811223. doi:10.1073/pnas.0801661105

Stoss O, Olbrich M, Hartmann AM, König H, Memmott J, Andreadis A, Stamm S. 2001. The STAR/GSG family protein rSLM-2 regulates the selection of alternative splice sites. J Biol Chem 276: 86658673. doi:10.1074/jbc.M006851200

Tilgner H, Nikolaou C, Althammer S, Sammeth M, Beato $M$, Valcárcel J, Guigó R. 2009. Nucleosome positioning as a determinant of exon recognition. Nat Struct Mol Biol 16: 996-1001. doi:10 $.1038 / \mathrm{nsmb} .1658$

Watermann DO, Tang Y, Zur Hausen A, Jäger M, Stamm S, Stickeler E. 2006. Splicing factor Tra2- $\beta 1$ is specifically induced in breast cancer and regulates alternative splicing of the CD44 gene. Cancer Res 66: 4774-4780. doi:10.1158/0008-5472.CAN-04-3294

Weinert BT, Narita T, Satpathy S, Srinivasan B, Hansen BK, Schölz C, Hamilton WB, Zucconi BE, Wang WW, Liu WR, et al. 2018. Timeresolved analysis reveals rapid dynamics and broad scope of the CBP/p300 acetylome. Cell 174: 231-244 e212. doi:10.1016/j .cell.2018.04.033

Xu Y, Gao XD, Lee JH, Huang H, Tan H, Ahn J, Reinke LM, Peter ME, Feng Y, Gius D, et al. 2014. Cell type-restricted activity of hnRNPM promotes breast cancer metastasis via regulating 


\section{Siam et al.}

alternative splicing. Genes Dev 28: 1191-1203. doi:10.1101/gad .241968 .114

Yang J, Weinberg RA. 2008. Epithelial-mesenchymal transition: at the crossroads of development and tumor metastasis. Dev Cell 14: 818-829. doi:10.1016/j.devcel.2008.05.009

Yang X, Coulombe-Huntington J, Kang S, Sheynkman GM, Hao T, Richardson A, Sun S, Yang F, Shen YA, Murray RR, et al. 2016. Widespread expansion of protein interaction capabilities by alternative splicing. Cell 164: 805-817. doi:10.1016/j.cell.2016 .01 .029
Yearim A, Gelfman S, Shayevitch R, Melcer S, Glaich O, Mallm JP, Nissim-Rafinia M, Cohen AH, Rippe K, Meshorer E, et al. 2015. HP1 is involved in regulating the global impact of DNA methylation on alternative splicing. Cell Rep 10: 1122-1134. doi:10 .1016/j.celrep.2015.01.038

Zhou HL, Hinman MN, Barron VA, Geng C, Zhou G, Luo G, Siegel RE, Lou H. 2011. Hu proteins regulate alternative splicing by inducing localized histone hyperacetylation in an RNA-dependent manner. Proc Natl Acad Sci 108: E627-E635. doi:10.1073/ pnas. 1103344108 

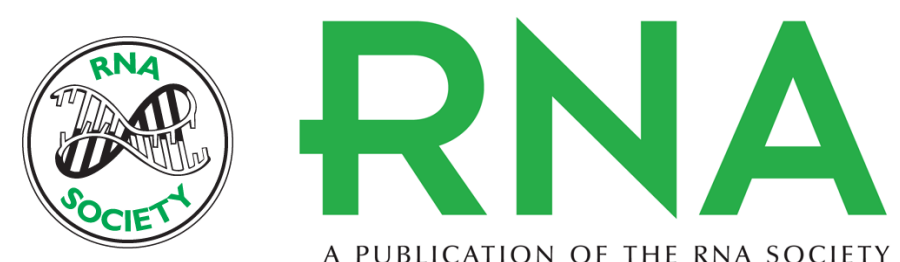

A PUBLICATION OF THE RNA SOCIETY

\section{Regulation of alternative splicing by $\mathrm{p} 300$-mediated acetylation of splicing factors}

Ahmad Siam, Mai Baker, Leah Amit, et al.

RNA 2019 25: 813-824 originally published online April 15, 2019

Access the most recent version at doi:10.1261/rna.069856.118

\section{Supplemental http://rnajournal.cshlp.org/content/suppl/2019/04/15/rna.069856.118.DC1 Material}

References This article cites 67 articles, 14 of which can be accessed free at: http://rnajournal.cshlp.org/content/25/7/813.full.html\#ref-list-1

Creative This article is distributed exclusively by the RNA Society for the first 12 months after the Commons License full-issue publication date (see http://rnajournal.cshlp.org/site/misc/terms.xhtml). After 12 months, it is available under a Creative Commons License (Attribution-NonCommercial 4.0 International), as described at http://creativecommons.org/licenses/by-nc/4.0/.

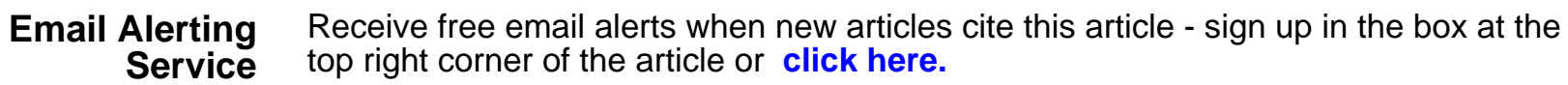

